\title{
Short-circuiting effect on the magnetoresistance of microstructured permalloy rings
}

\author{
C.T. Chao ${ }^{\text {a }}$, L.K. Lin ${ }^{\mathrm{a}}$, J.C. Wu ${ }^{\mathrm{a}, *}$, Zung-Hang Wei ${ }^{\mathrm{b}}$, Mei-Feng Lai ${ }^{\mathrm{c}}$, Ching-Ray Chang ${ }^{\mathrm{d}}$ \\ ${ }^{a}$ Department of Physics, National Changhua University of Education, Changhua 500, Taiwan \\ ${ }^{\mathrm{b}}$ Department of Power Mechanical Engineering, National Tsing Hua University, HsinChu 300, Taiwan \\ ${ }^{c}$ Department of Engineering Science and Ocean Engineering, National Taiwan University, Taipei 10617, Taiwan \\ ${ }^{\mathrm{d}}$ Department of Physics, National Taiwan University, Taipei 10617, Taiwan
}

Available online 27 November 2006

\begin{abstract}
We present a systematic investigation of the current/voltage lead effect on magnetization reversal of microstructured permalloy rings. The magnetic rings with film thickness of $23 \mathrm{~nm}$ and outer/inner diameter of $5 / 3 \mu \mathrm{m}$, respectively, were patched by nonmagnetic gold leads designed with various angles, $10^{\circ}, 20^{\circ}$, and $30^{\circ}$. Longitudinal magnetoresistance measurement was adopted to demonstrate the short-circuiting effect. The mechanism can be attributed to the formation of the vortex structures developed beneath the lead-covered areas, in which the vortex pair domain configuration was confirmed using a magnetic force microscopy.
\end{abstract}

(C) 2006 Elsevier B.V. All rights reserved.

PACS: 75.06.-d; 70.50.Cc

Keywords: Permalloy ring; Magnetoresistance; Domain wall; Vortex

Magnetic ring structures have been of great interest due to potential application for magnetic random access memories (MRAM) cells [1]. The advantages of using such annular shape are as follows. The ring structure easily forms stable flux-closure magnetization, thus prevents magnetic field from leaking out and reduces the influence between adjacent memory cells. As a result, studies of magnetization reversal process and the manipulation of magnetization [2,3] using electric measurements and magnetic force microscope (MFM) are the priority before implementing into real devices. However, among the magnetoresistance measurements reported in the past no concern has been paid to the effect associated with the nonmagnetic current/voltage leads. In our previous paper [4], an electrical shorting behavior resulted from the gold current/voltage leads was for the first time reported. Herein, we present a systematic study on the influence of the nonmagnetic leads by varying the patching areas, from

\footnotetext{
${ }^{*}$ Corresponding author. Tel.: + 8867253896 ; fax: +8867253896 .

E-mail address: phjcwu@cc.ncue.edu.tw (J.C. Wu).
}

which the degree of shorting effect can be seen from the MR curves and furthermore one may be able to estimate the size of the vortex.

The ring devices were designed with outer/inner diameter $5 / 3 \mu \mathrm{m}$ using standard electron beam lithography. The permalloy thin film was thermally evaporated in the absence of any external magnetic fields. The nonmagnetic gold leads were patched on the permalloy rings by another step of electron beam lithography and designed with angles of $10^{\circ}, 20^{\circ}$, and $30^{\circ}$, respectively. Finally, a four-terminal DC measurement was carried out with the external magnetic field applied along the current direction, a schematic diagram is shown in Fig. 1. It has been known that the magnetoresistance of permalloy thin film depends on the angle between current and magnetization direction, which is called anisotropic magnetoresistance (AMR) effect. In our previous finding [4], it was shown that the magnetization of ring devices evolved from onion state at the saturation field to vortex pair state at the near zero field. Consequently, the formation of the vortex domain wall gives rise to the ring resistance change in low field, 


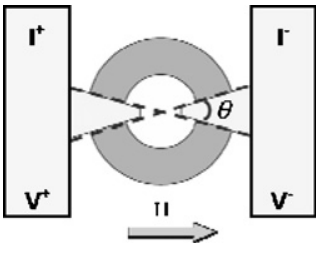

Fig. 1. The schematic diagram shows the device with nonmagnetic current/voltage leads. The external field was applied along the current direction.

a

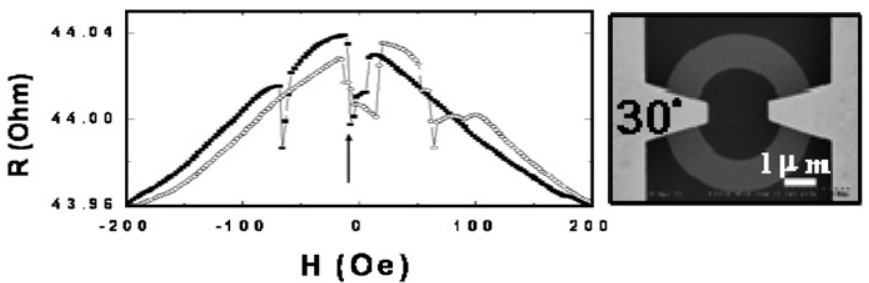

b

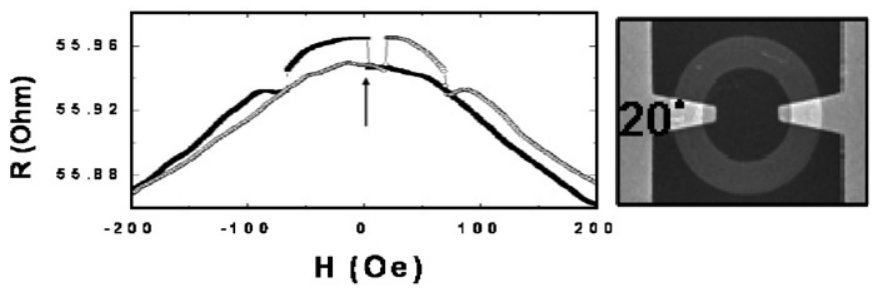

C

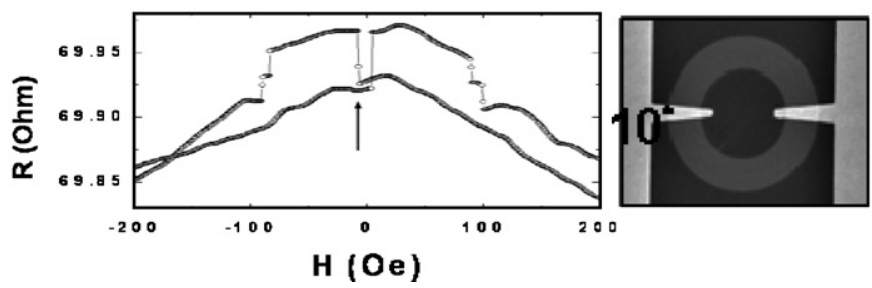

Fig. 2. A series of magnetoresistance curves measured on the permalloy rings having patching angles of (a) $30^{\circ}$, (b) $20^{\circ}$, and (c) $10^{\circ}$, respectively. The scanning electron micrographs shown alongside are the corresponding devices, in which the brighter areas are nonmagnetic gold leads. Notice that the arrows indicate the resistance drops argued for the electrical shorting effect in the text.

provided that the patching area is smaller than the size of vortex.

Fig. 2 shows a series of MR curves measured on the permalloy ring devices with various patching angles of nonmagnetic gold leads. Through the MR curves, two transitions in $H=0$ and -70 Oe during the switching process can be identified. Based on AMR effect, the plateau between two transitions corresponds to a vortex configuration in the ring. Magnetization reversals revealed in the MR curves are all similar, however significant resistance drops manifest at the transitions near zero field with larger leads angle of devices, as shown in Fig. 2(a), and become less discernable in the smaller leads angle of devices, as shown in Fig. 2(b) and (c).

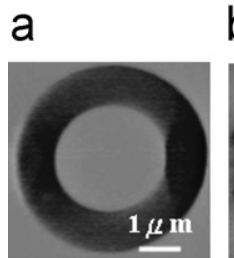

$\mathrm{H}=162 \mathrm{Oe}$
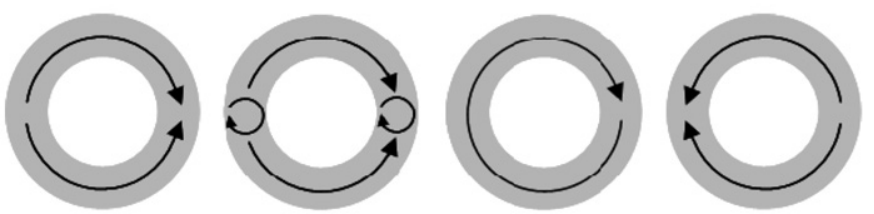

Fig. 3. A series of MFM images are shown in the upper row and the corresponding schematics are illustrated in the lower row. The MFM images from left to right are taken in the presence of different external fields: (a) $162 \mathrm{Oe}$, (b) $0 \mathrm{Oe},(\mathrm{c})-11 \mathrm{Oe}$, and (d) $-76 \mathrm{Oe}$, respectively. Note that a magnetization transition occurs at the near zero field during the sweep-down process (onion to vortex pair).

A series of MFM images shown in the upper row of Fig. 3 reveal (from left to right) onion, vortex-pair, vortex, and reversed onion state with the schematics shown in the lower row of Fig. 3. The external fields corresponding to the aforementioned magnetization configurations have a good agreement with those measured for the MR curves based on the AMR effect, as argued in our previous paper [4]. In comparison to the resistance drop due to vortex pair formation, as shown in Fig. 3(b), the larger angles of gold leads fully cover the vortex domain wall structures and give rise to more significant drops in resistance, as shown in Fig. 2(a). The nonmagnetic gold leads shunt the segment of the permalloy ring underneath. These phenomena were indeed further identified with the transverse MR measurements, where vortex domain wall structures formed in the opposite ends encounter no gold leads, thus giving rise to no resistance drops at the transition fields, as shown elsewhere [4].

In summary, we have shown a short-circuiting effect on MR measurements of the microstructured permalloy rings patched with nonmagnetic gold leads. The ring devices were designed to have magnetization reversal from onion at the saturation field to vortex pair state at the near zero field causing resistance drops based on AMR effect. In addition, the nonmagnetic gold leads shunt the segment of the permalloy ring underneath. Here, we have shown that the short-circuiting effect from gold leads dominates when they fully cover the areas of the vortex walls. This result may provide important information in designing devices for real applications.

This work was supported by the Ministry of Economic Affairs of Taiwan with grant no. 94-EC-17-A-01-S1-026 
and the National Science Council of Taiwan with grant no. NSC94-2112-M-018-002.

\section{References}

[1] J.-G. Zhu, Y. Zheng, G.A. Prinz, J. Appl. Phys. 87 (2000) 6668.

[2] M. Kläui, C.A.F. Vaz, J.A.C. Bland, T.L. Monchesky, J. Unguris, E. Bauer, S. Cherifi, S. Heun, A. Locatelli, L.J. Heyderman, Z. Cui, Phys. Rev. B 68 (2003) 134426.
[3] T. Uhlig, J. Zweck, Phys. Rev. Lett. 93 (2004) 047203.

[4] M.-F. Lai, Z.-H. Wei, C.-R. Chang, J.C. Wu, J.H. Kuo, J.-Y. Lai, Phys. Rev. B 67 (2003) 104419. 